\title{
A Fast Algorithm for Matching Planar Maps with Minimum Fréchet Distances
}

\author{
Junichi Shigezumi \\ Fujitsu Laboratories Ltd. \\ 1-1, Kamikodanaka 4-chome \\ Nakahara-ku, Kawasaki \\ 211-8588, Japan \\ j.shigezumi@jp.fujitsu.com \\ Hiroaki Morikawa \\ Fujitsu Laboratories Ltd. \\ 1-1, Kamikodanaka 4-chome \\ Nakahara-ku, Kawasaki \\ 211-8588, Japan \\ h.morikawa@jp.fujitsu.com inakoshi.hiroya@jp.fujitsu.com \\ Tatsuya Asai \\ Fujitsu Laboratories Ltd. \\ 1-1, Kamikodanaka 4-chome \\ Nakahara-ku, Kawasaki \\ 211-8588, Japan \\ asai.tatsuya@jp.fujitsu.com \\ Hiroya Inakoshi \\ Fuijtsu Laboratories Ltd. \\ 1-1, Kamikodanaka 4-chome \\ Nakahara-ku, Kawasaki \\ 211-8588, Japan
}

\begin{abstract}
In this paper, we present a fast and practical algorithm for a map-matching problem searching a path on a given graph that minimizes Fréchet distance from a given trajectory, which is a natural measurement based on the sequential order of the trajectory. However, it sometimes costs seriously to compute the Fréchet distance while making correspondences to on a path on the graph in the order from the beginning of the trajectory as a naive method (as the definition) since it often occurs to backtrack and recompute. We developed an incremental technique for updating the Fréchet distance between the trajectory and a path to overcome the problem stated above. It enables the proposed algorithm to evaluate distances for any candidate paths faster than the naive one. In addition, we can adopt Dijkstra's graph searching manner due to the technique and omit to search and evaluate some useless candidates which have no relations with the solution. That also contributes to accelerate the algorithm. Experimental results show that our algorithm was more than fifty times faster than the algorithm of Alt (J. Algorithms 2003), which is formulated as a optimization problem repeating to solve decision problems with a binary search on a set of candidates of the Fréchet distance.
\end{abstract}

\section{Categories and Subject Descriptors}

H.2.8 [Database Applications]: Spatial Databases and GIS

\section{General Terms}

Algorithms, Experimentation

Permission to make digital or hard copies of all or part of this work for personal or classroom use is granted without fee provided that copies are not made or distributed for profit or commercial advantage and that copies bear this notice and the full citation on the first page. To copy otherwise, to republish, to post on servers or to redistribute to lists, requires prior specific permission and/or a fee.

ACM SIGSPATIAL International Workshop on Analytics for Big Geospatial Data 2015, Seattle, WA, USA

Copyright 2015 ACM 978-1-4503-3974-2 ...\$15.00.

DOI: $10.1145 / 2835185.2835186$

\section{Keywords}

Map-matching, Fréchet distance, trajectory, road network

\section{INTRODUCTION}

With the development of technology and a number of lowcost GPS-equipped devices, a massive amount of location information has been collected [27]. GPS receivers are widely used in aircraft, ships, and motor vehicles for both business and personal use. They are extensively used for fleet management and asset tracking in the logistics and retail industries [11]. The development of applications and services for collecting location information from GPS devices as well as from other sources such as global systems for mobile communication and Wi-Fi networks enables people to share their experiences with friends through location histories.

Furthermore, with trajectories that are sequences of locational positions collected by GPS, we can track each movement of a person or an object. However, the raw data has uncertainty caused by measurement errors from GPS accuracy and sampling errors from the sampling rate [21]. These factors make it difficult to identify trajectories.

For this purpose, several map-matching methods that search a path on a given graph, representing a road map or a more abstract map, use a small distance from a given trajectory. These are presented in $[3,5,6,14,15,16,17,19,22]$. Furthermore, other related methods have been proposed that consolidate similar trajectories by using the distance of curves [1, 7, 8, 31].

The Fréchet distance is one such distance measure for curves.

Definition 1. (Fréchet distance [13,2]) The Fréchet distance between two curves, $f: I=\left[l_{I}, r_{I}\right] \rightarrow \mathbb{R}^{2}$ and $g: J=$ $\left[l_{J}, r_{J}\right] \rightarrow \mathbb{R}^{2}$, is defined as:

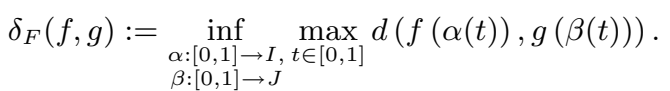

Here, the functions $\alpha$ and $\beta$ are monotonically increasing 
continuous functions that satisfy $\alpha(0)=l_{I}, \alpha(1)=r_{I}$, $\beta(0)=l_{J}$, and $\beta(1)=r_{J}$. We denote the Euclidean distance by $d: \mathbb{R}^{2} \times \mathbb{R}^{2} \rightarrow \mathbb{R}_{\geq 0}$.

The most important property of the Fréchet distance is monotonicity of the functions $\alpha$ and $\beta$. The property is related to the sequential orders of the points on trajectories (or the directions of the trip).

The Fréchet distance is a natural distance measure for comparing curves. In general, the trajectory data may contain complex movements such as loops or U-turns, and so may the structure of road maps. If we consider only the distances between the points of the trajectory and paths, we cannot measure the similarity between them accurately. The Fréchet distance is more appropriate than other distance measures like the Hausdorff distance [4]. By considering the sequential order of trajectories, we can obtain better similarity between them by using the Fréchet distance. [3, 6]

There are two main problems with researching problems that involve the Fréchet distance of curves. The first is a decision problem, which involves deciding whether the distance of curves is at most a given threshold $\varepsilon$ or not. The second is an optimization problem, which involves solving the Fréchet distance of curves.

Similarly, we obtain two problems for graph search problems. For the decision problem on a graph, we search a path whose distance from a trajectory is at most a given threshold $\varepsilon$. For the optimization problem on a graph, we search a path that minimizes the distance from the trajectory.

Alt et al. [3] presented an algorithm for the decision problem in $O(p q \log p)$ time, where we denote $p$ and $q$ as the length of the trajectory and the size of the graph, respectively. Furthermore, they present a method for solving the optimization problem in $O(p q \log (p q) \log p)$ time by using an application of the above algorithm. This involves executing $O(\log (p q))$ number of iterations of the algorithm of their decision problem. For the optimization problem, some approximation approaches were presented [6, 12, 18, 25].

In this paper, we study algorithms for the optimization problem on a graph. The algorithms presented by Alt et al. [2,3] are theoretically efficient. However, if the target is the optimization problem, then it is natural to directly construct an algorithm for the problem. Then, we present a fast and practical algorithm for a map-matching problem that searches a path on a given graph that minimizes the Fréchet distance from a given trajectory, which is a natural measurement based on the sequential order of the trajectory.

If we compute the Fréchet distance while making correspondences to a path on a graph in the order from the beginning of the trajectory as a naive method, backtracking and recomputing often occurs, which sometimes has serious computational cost.

The contributions of this paper are described as follows.

- We developed an incremental technique for updating the Fréchet distance between the trajectory and a path by combining "critical values" (see Section 4). The technique enables the proposed algorithm to evaluate distances for any candidate paths faster than the naive one.

- In addition, we can adopt Dijkstra's graph searching manner with the technique and omit searching and evaluating useless candidates that have no relation to the solution. This also contributes to accelerating the algorithm.

As a result, experimental results show that our algorithm is more than fifty times faster than that of Alt et al. [3], which uses a binary search on a set of candidates of the Fréchet distance.

With our algorithm of map-matching with Fréchet distance, we can translate from raw GPS data with errors into smooth ways on the road networks. In addition, as a future work, we would like to match a trajectory to other trajectories in a dataset, for searching a similar person or constructing taxi-sharing systems. It may take much cost for the huge dataset. Then, our fast algorithm will work well.

\section{PRELIMINARIES}

In this section, we give basic notations and definitions. A trajectory is a sequence of points on a two-dimensional Euclidean space $\mathbb{R}^{2}$.

A graph $G$ is defined as a pair $(V, E)$ comprising a set of vertices $V$ and a set of edges $E$. In this paper, we deal with a geometric graph (in short, graph), whose vertices are points of $\mathbb{R}^{2}$.

Furthermore, a curve consisting of a sequence of points and line segments connecting consecutive points is called a "polygonal curve" (in short, curve). For a sequence of points $\left\{x_{0}, \ldots, x_{p}\right\}$, we can naturally construct a polygonal curve $f: I=[0, p] \rightarrow \mathbb{R}^{2}$; we have $f(i)=x_{i}$ for each integer $0 \leq i \leq p$, and $f([i, i+1])$ is a line segment connecting points $x_{i}$ and $x_{i+1}$. We also denote $|f|:=p$ as the length of the trajectory $f$.

We regard trajectories and paths on graphs as polygonal curves. Then, we measure the distances between a trajectory and a path on a graph.

In this paper, we consider the following problem.

Problem 1. (Optimization problem on a graph) For a given trajectory and a graph, search a path on the graph that minimizes the Fréchet distance from the trajectory.

Figure 1 gives an example of a path on the graph that minimizes the Fréchet distance from the trajectory.

\subsection{On map matching with the Fréchet dis- tance}

The Fréchet distance is a natural distance measure for comparing curves. It is generally said to be more appropriate 


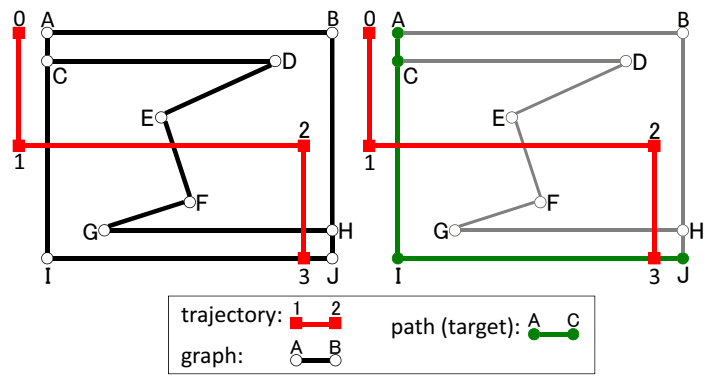

Figure 1: Example of trajectory, geometric graph (left), and a path (right) on the graph that minimizes the Fréchet distance from the trajectory

for distance than the Hausdorff distance or other distances [4].

In Figure 1, we select path $p_{0}:=\langle A, C, I, J\rangle$, which minimize the Fréchet distance from a given trajectory. With the Hausdorff distance, we have another path

$p_{1}:=\langle A, C, D, E, F, G, H, J\rangle$. Comparing the two paths, path $p_{0}$ has a smaller Fréchet distance from the trajectory since $p_{1}$ moves back and forth. It gives us a more natural result of map-matching.

Figure 3 shows another example of actual data (T-drive, see Section 7.1). The trajectory and the map in the figure are very difficult for map-matching; the trajectory has a long interval and positioning errors, and the map has roads that run closely to and in parallel on both sides of the river. Thus, by map-matching with Hausdorff distance, we chose a very complex path on the road map, where distances between points on the trajectory and the path are small, but the path contains three loops. In comparison, by map-matching with the Fréchet distance, we could choose a very smooth way. With the Fréchet distance, we can evaluate not only the distances between points but also the differences of directions in such loops.

\section{RELATED WORK}

Many topological map-matching algorithms were introduced. White et al. [26] studied map-matching algorithms for inaccurate networks. They present algorithms with different type of matching, that is, point-to-point, point-to-curve, and curve-to-curve matching. Their most advanced algorithm with curve-to-curve matching uses a local measure of the distances of curves, although the Fréchet distance is a global measure of the distances of curves. Greenfeld [15] presents a map-matching algorithm for GPS data and digital map with inaccuracies and errors. The algorithm is based on distance and orientation, and it uses a greedy strategy for massive incoming data. He uses local similarity for the greedy incremental method. Quddus et al. [22] developed map-matching algorithms based on various similarities. They perform efficiently for junctions and intersections if we select the proper parameters of weighting factors.

However, as introduced above, they are incremental methods with local similarity. Brakatsoulas et al. [6] studied three algorithms. The first is an incremental algorithm with local similarity, the second is a global algorithm [3] with the Fréchet distance, which is a global measure, and the third is a global algorithm with weak Fréchet distance. The global algorithms produced better matching results, although the incremental algorithm ran faster.

Another type of map-matching algorithms was introduced by Lou et al. [17] and Newson et al. [19] based on hidden Markov models, which are the statistical models. They presented map-matching algorithms for low sampling and noisy trajectory data. It is important future work to study the differences between topological and statistical map-matching methods.

\section{THE ALGORITHM OF ALT ET AL.}

In this section, we present a summary of the algorithms presented by Alt et al. [2, 3]. Furthermore, we explain important notations and properties for computing Fréchet distances, which is key in the implementation of our algorithm described in Section 6. We can refer to both Alt et al. [2] and Alt et al. [3] for computing of Fréchet distances. Particularly, in Alt et al. [3], they apply their method to polygonal curves (Alt et al. [2]) to graph search problems.

\subsection{Alt's algorithms for the decision problem}

In Alt et al. [2], they introduce free space diagrams to solve the decision problem on curves with a threshold $\varepsilon$, where we decide whether the Fréchet distance between two given curves is at most $\varepsilon$ or not. Figure 2 shows the curves $f, g$ and a corresponding free space diagram with a threshold $\varepsilon$. In the diagram, on the white point $(x, y)$, the distance of the points $f(x)$ and $g(y)$ is at most $\varepsilon$. Conversely, on the black point, the distance of the corresponding points on the curves $f$ and $g$ is greater than $\varepsilon$.
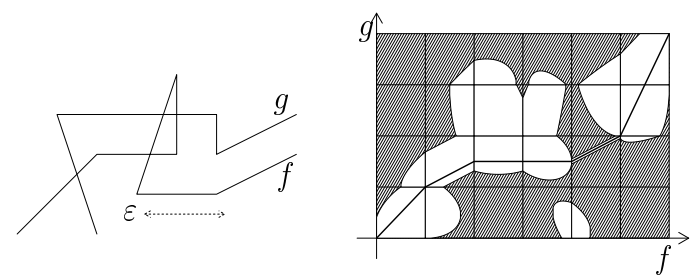

Figure 2: Free space diagram (right) for the curves $f$ and $g$ (left) with a threshold $\varepsilon$ [2]

Now, if we can draw a monotone curve on a connected white area in the free space diagram, as shown in Figure 2, we may define monotone functions $\alpha$ and $\beta$ with Definition 1 , which satisfies $d(f(\alpha(t)), g(\beta(t))) \leq \varepsilon$. We call such a curve a feasible curve. Then, the Fréchet distance between curves $f$ and $g$ is at most $\varepsilon$ if and only if we have a feasible curve on the free space diagram with threshold $\varepsilon$.

Furthermore, we only have to check white intervals on the grid in the free space diagram (exact notation is shown in the next paragraph) to know whether we have a feasible curve or not. This derives from the convexity of the white area in each mesh of the grid.

For the white intervals, we use the following notations (see Figure 4). Let $i$ and $j$ be integers that satisfy $0 \leq i \leq|f|$ 

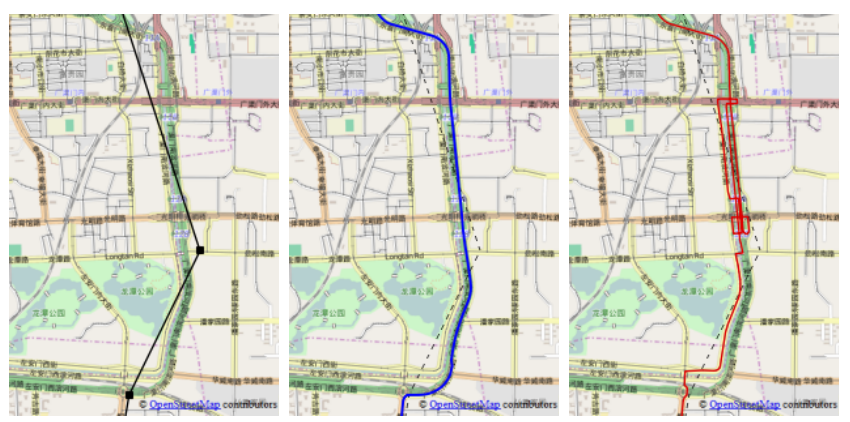

(Maps data from (C)OpenStreetMaps ${ }^{1}$ contributors)

Figure 3: Example of a trajectory (left) and map-matching of it with the Fréchet distance (center) and Hausdorff distance (right)

and $0 \leq j \leq|g|$. We denote white intervals as $L_{i j}:=\{j \leq$ $y \leq j+1: d(f(i), g(y)) \leq \varepsilon\}$ and $B_{i j}:=\{i \leq x \leq i+$ $1: d(f(x), g(j)) \leq \varepsilon\}$. Furthermore, if we have $L_{i j} \neq \phi$ and $B_{i j} \neq \phi$, we write $L_{i j}=\left[a_{i j}, b_{i j}\right]$ and $B_{i j}=\left[c_{i j}, d_{i j}\right]$, respectively. Conversely, if we have $L_{i j}=\phi$ and $B_{i j}=\phi$, we define $\left(a_{i j}, b_{i j}\right)=(j+1, j)$ and $\left(c_{i j}, d_{i j}\right)=(i+1, i)$ for convenience, respectively.

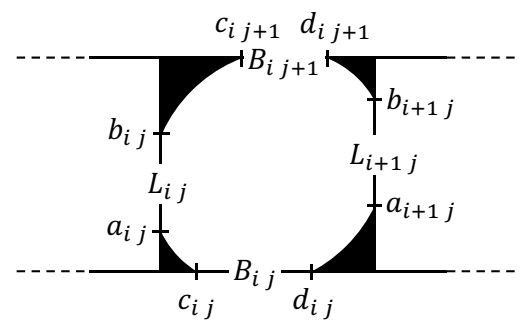

Figure 4: Notations on a free space diagram [2]

Alt et al. [3] developed the above-mentioned method into a decision problem on a graph with a threshold $\varepsilon$, where we search a path on a given graph whose Fréchet distance from a given trajectory is at most $\varepsilon$. Figure 5 shows the free space surface for a graph, which corresponds to the free space diagram for the curves. Similar to the case of curves, if we have a feasible curve on the free space surface with threshold $\varepsilon$, then we have a solution, which is a path on the graph whose Fréchet distance from the trajectory is at most $\varepsilon$.

\subsection{Alt's algorithms for the optimization prob- lem}

For the optimization problem, in Alt et al. [2], they proposed the following method, which applies the algorithm to the decision problem.

On a free space diagram, the white area becomes smaller as the threshold $\varepsilon$ decreases. Similarly, the white intervals become smaller. At the moment when the threshold becomes smaller than the Fréchet distance, some white points on feasible curves change to black. Alt et al. [2] noted such points and stated that this occurs under the following three
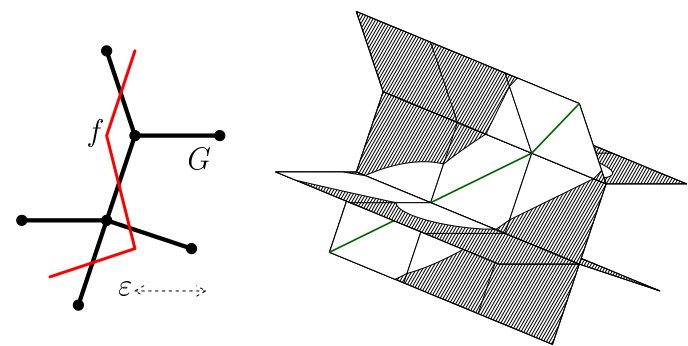

Figure 5: Free space surface (right) for the trajectory $f$ and the graph $G$ (left) with a threshold $\varepsilon$

conditions. They defined critical values as corresponding thresholds $\varepsilon$.

\section{Condition 4.2.1}

a) We have $(i, j)=(0,0)$ or $(i, j)=(p, q)$.

b) For some integers $i, j$, we have $a_{i j}=b_{i j}$ or $c_{i j}=d_{i j}$.

c) For some integers $i, j, k$, we have $a_{i j}=b_{k j}$ or $c_{i j}=d_{i k}$.

Condition a) corresponds to the first points or the last points of the curves. Condition b) occurs when one of the vertices of a curve corresponds to a point on one of the line segments of the other curve. Condition c) occurs when two distinct vertices of a curve correspond to a point on one of the line segments of the other curve. (See Fig 6.)

Note that the Fréchet distance coincides with one of the critical values. Thus, we can search the Fréchet distance from the set of critical values.

Then, we use the algorithm for the decision problem described in Section 4.1. If the selected critical value $\varepsilon$ is at least the Fréchet distance, we then have a solution for the decision problem with the threshold $\varepsilon$. After iterations for selecting the critical values and executions of the algorithm for the decision problem, we get a critical value equal to the Fréchet distance.

In conclusion, Alt et al. [2, 3] constructed the algorithm for the decision problem and applied it to the optimization problem. Their algorithms are theoretically efficient. However, if the target is the optimization problem, it is natural to directly construct an algorithm for the problem. In this paper, we present a fast and practical algorithm for the optimization problem with a property of critical values.

\subsection{A property of critical values}

One of the most important properties of critical values is the third condition, condition c) in 4.2.1, which is a base of efficiency of the algorithms of Alt et al. [2,3] and our algorithm proposed in Section 6 .

For the case of $a_{i j}=b_{k j}$ in condition c), the $i$ th and $k$ th points of the curve $f$ correspond to the $j$ th segment of $g$. 

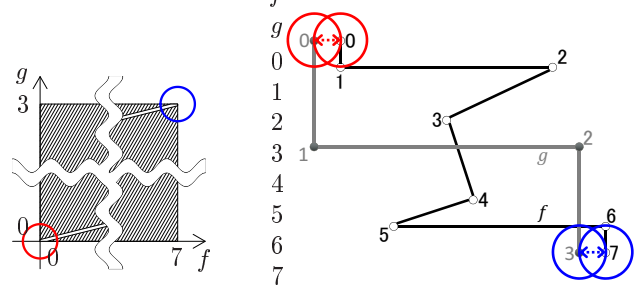

a) $(i, j)=(0,0)$ (left), $(7,3)$ (right)
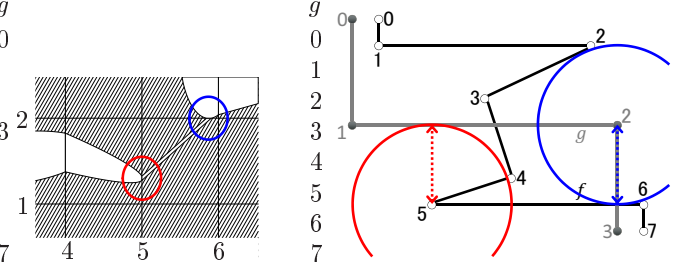

b) $a_{51}=b_{51}$ (left), $c_{52}=d_{52}$ (right)

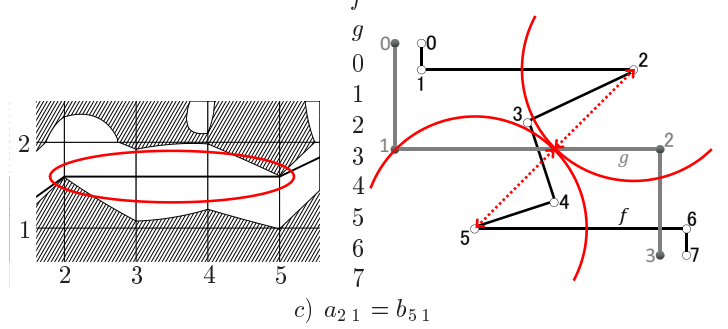

Figure 6: Examples for each condition in 4.2.1: Figures show the free space diagrams (left) and curves of $f$ and $g$ (right). In each diagram, each equality of the condition holds at the point in the circles. In the figures of curves, each arrow corresponds to the point where each equality holds, and then the lengths of arrows (or radius of circles) are critical values.

However, if we have a feasible curve on a corresponding white area, we must check the white intervals on all of the $l$ th points $(i \leq l \leq k)$ and $j$ th segments. Thus, we have to show that

$$
\max _{i \leq l^{\prime} \leq l} a_{l^{\prime}, j} \leq \min _{l \leq l^{\prime \prime} \leq k} b_{l^{\prime \prime}, j}
$$

Furthermore, since the behaviors of the values $a_{l, j}$ and $b_{l, j}$ under the value $\varepsilon$ are different from each $l$, it is difficult to consider all the combinations of all of the $l$ th white intervals.

However, practically, we only have to check every pair in condition c). For example, we can select $l^{\prime}$ and $l^{\prime \prime}$ on each side of inequality (2) where two sides are equal. Thus, when we compute all of the values $\varepsilon$ that satisfy $a_{l^{\prime}, j}=b_{l^{\prime \prime}, j}$ for a pair of $l^{\prime}$ and $l^{\prime \prime}$ with $i \leq l^{\prime} \leq l^{\prime \prime} \leq k$, one of the values $\varepsilon$ corresponds to the value where both sides of (2) are equal.

Although Condition 4.2.1 is defined with the notation of white intervals, we can also define the critical values without white intervals as follows.

Critical values corresponding to Condition 4.2.1

i) $\varepsilon_{(i, k),\left(j, j^{\prime}\right)}^{L}$ $:=\min _{j \leq y_{1} \leq y_{2} \leq j^{\prime}} \max \left\{d\left(f(i), g\left(y_{1}\right)\right), d\left(f(k), g\left(y_{2}\right)\right)\right\}$

ii) $\varepsilon_{\left(i, i^{\prime}\right),(j, k)}^{B}$

$$
\stackrel{:=\min _{i \leq x_{1}} \leq x_{2} \leq i^{\prime}}{\max }\left\{d\left(f\left(x_{1}\right), g(j)\right), d\left(f\left(x_{2}\right), g(k)\right)\right\}
$$

The above two cases contain all three cases of Condition 4.2.1. For condition a), the case of $(i, j)=(0,0)$ and $(i, j)=$ $(p, q)$ corresponds to $\varepsilon_{(0,0),(0,0)}^{L}=d(f(0), g(0))$ and $\varepsilon_{(p, p),(q, q)}^{L}=$ $d(f(p), g(q))$. For condition b), the case of $a_{i j}=b_{i j}$ and $c_{i j}=d_{i j}$ corresponds to $\varepsilon_{(i, i),(j, j+1)}^{L}$ and $\varepsilon_{(i, i+1),(j, j)}^{B}$. For condition c), the case of $a_{i j}=b_{k j}$ and $c_{i j}=d_{i k}$ corresponds to $\varepsilon_{(i, k),(j, j+1)}^{L}$ and $\varepsilon_{(i, i+1),(j, k)}^{B}$. The superscripts " $L$ " and " $B$ " correspond to the notations $L_{i, j}$ and $B_{i, j}$.

\section{COMPUTING THE FRÉCHET DISTANCE}

In this section, we propose an incremental technique for updating the Fréchet distance between a trajectory and a path. It enables us to construct an algorithm to solve the optimization problem directly.

Alt et al. [2, 3] constructed efficient algorithms for the optimization problem. Their algorithms repeat to solve the number of decision problems, where we can only decide whether a distance is greater than the given thresholds or not. However, considering the definition of the optimization problem, it should be natural to search a path directly by comparing the distances of candidates. However, there have been no such algorithms that can do so. The reason is the difficulty of updating the Fréchet distance, which requires strict correspondence between a trajectory and a path.

In Definition 1, a Fréchet distance is the maximum of distances between points that are associated with each other by the functions $\alpha$ and $\beta$. Then, in a natural way, after determining the two functions, we can compute the distances between points on two curves. However, it often requires much computational cost to determine the function in each step of graph search problems. For example, incremental searches on a graph need to update the function while searching, but this is not easy. We possibly have to determine functions all over again from the beginning.

Figure 7 shows an example of Fréchet distances on steps in a graph search problem. Each dotted line connects points on curves $f$ and $g$, which correspond to each other with functions $\alpha$ and $\beta$. Except for the beginning points, almost all of the correspondences between the two curves are different in each step $i, i i$, and $i i i$.

However, all we want to do is not to determine the functions but to compute the Fréchet distances. Thus, the property of the critical values in the previous section is also effective for our algorithm because we only have to compute every additional pair of vertices of a graph or the points of a trajectory.

For a graph search problem, we use the following notations for critical values.

Critical values for graph search problem

i) $\varepsilon_{(i, k),\left(v, v^{\prime}\right)}^{L}:=\min _{0 \leq r_{1} \leq r_{2} \leq 1}$ $\max \left\{d\left(f(i), r_{1} v+\left(1-r_{1}\right) v^{\prime}\right), d\left(f(k), r_{2} v+\left(1-r_{2}\right) v^{\prime}\right)\right\}$, 


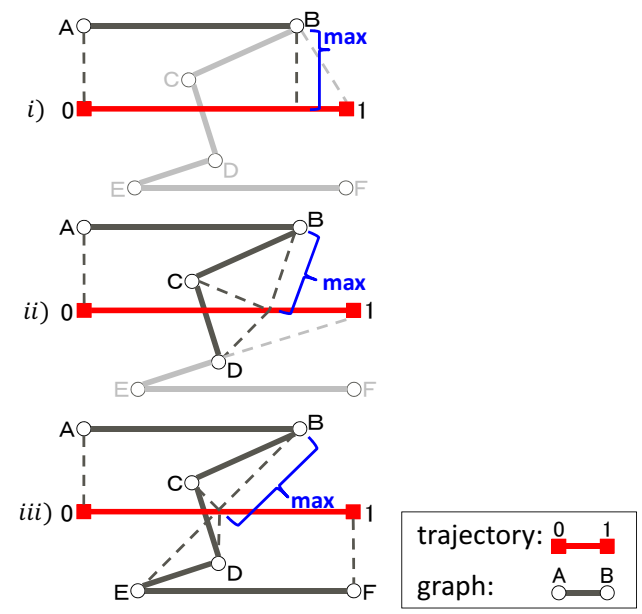

Figure 7: Examples of updating Fréchet distances with incremental graph searching

ii) $\varepsilon_{\left(i, i^{\prime}\right),(v, w)}^{B}$

$$
:=\min _{i \leq x_{1} \leq x_{2} \leq i^{\prime}} \max \left\{d\left(f\left(x_{1}\right), v\right), d\left(f\left(x_{2}\right), w\right)\right\} .
$$

In Figure 7, the Fréchet distance of each steps $i, i i$, and $i i i$ is $\varepsilon_{(0,1),(B, B)}^{B}, \varepsilon_{(0,1),(B, C)}^{B}$, and $\varepsilon_{(0,1),(B, E)}^{B}$, respectively. In fact, from step $i$ to step $i i$, we take the maximum of $\varepsilon_{(0,1),(B, B)}^{B}$ and the critical values of additional pairs $\varepsilon_{(0,1),(A, C)}^{B}, \varepsilon_{(0,1),(B, C)}^{B}$ $\varepsilon_{(0,1),(A, D)}^{B}, \varepsilon_{(0,1),(B, D)}^{B}$, and $\varepsilon_{(0,1),(C, D)}^{B}$. Then, we obtain the distance $\varepsilon_{(0,1),(B, C)}^{B}$ for step $i i$. This is an example of updating distances for additional pairs of the vertices of a graph. We can consider updating distances for the additional pairs of the points of a trajectory in the same way.

Critical values were introduced in Alt et al. [3] only as candidates of the Fréchet distance. However, by using the critical values as in this section, we can compute and update the Fréchet distance directly.

\section{PROPOSED ALGORITHM}

In this section, we present an algorithm for the optimization problem on a graph defined as Problem 1.

We propose a Dijkstra-type algorithm with a priority that is the Fréchet distance between a candidate path and the corresponding part of the trajectory. The key of our algorithm is how we compute and update the Fréchet distance. We make it possible to compute the distance with the property of the critical values, which are discussed in Section 4.3.

This section is organized as follows. In Section 6.1, we provide the architecture of the proposed algorithm. In Section 6.2 , we introduce a model to update the distances, and finally, in Section 6.3, we describe an improvement to the algorithm.

\subsection{Architecture of the proposed algorithm}

The proposed method follows a Dijkstra-type algorithm [10]. The Dijkstra-type algorithm is based on a best first searching strategy, where we can omit searching and evaluating useless candidates that have no relation to the solution. It also accelerates the algorithm.

In general, the Dijkstra algorithm is a graph search algorithm for finding the shortest path in a directed graph with weighted edges. A priority is defined as a function that monotonically increases as the search steps progress in the Dijkstra algorithm [10]. We have the following iteration. First, we select a candidate that minimizes the priority. Then, we proceed to the neighborhoods of the current vertex corresponding to the selected candidate and then update the priority. Finally, we add it as a new candidate. At the end of the iterations, we obtain a path that minimizes the priority.

The key to designing a Dijkstra-type algorithm is the priority. With Dijkstra's algorithm for computing the shortest path, there are non-negative weights on each edge, and we define the priority as a summation of the weights of edges on the path. Following the search steps, the value of priority monotonically increases with the addition of non-negative values. Thus, we can obtain a path that minimizes the priority, which is a shortest path.

In comparison, in our algorithm, we define the priority as the Fréchet distance between a candidate path and the corresponding part of the trajectory. Thus, we can obtain a path that minimizes the Fréchet distance with the trajectory. We selected the Dijkstra-type algorithm, where we have to update distances only for the prior candidate in each step. Then, we can omit searching and evaluating useless candidates that have no relation to the solution except for the worst case scenarios.

Algorithm 1 shows the entire flow of our algorithm. First, for all the vertices in the graph, we compute distances from the starting point of the trajectory and add them as initial candidates (lines $2-5$ ). Next, we choose a candidate with the minimum Fréchet distance corresponding to the maximum index of the trajectory (line 7). After that, we perform the search step with the neighbors of the current vertex (line 12) and then update the Fréchet distance (line 13). When the selected prior path corresponds to the end point of the trajectory, we finish the search (line 10) and return the path.

\subsection{Updating distances}

In this section, we introduce the updating of the Fréchet distances between a candidate path and the corresponding parts of the trajectory, which is the key procedure of the overall algorithm.

We compute and update the Fréchet distances between a candidate path and the corresponding parts of the trajectory. Algorithm 2 shows this procedure.

As mentioned in Section 5, we only have to compute critical values for each additional pair in order to compute and update the Fréchet distances. In Algorithm 2, we update distances with the critical values of the additional pairs of the points of the trajectory (lines $4-6$ ) and those of additional pairs of the vertices of the graph (lines $8-10$ ).

In the above algorithm, we compute distances for every point 

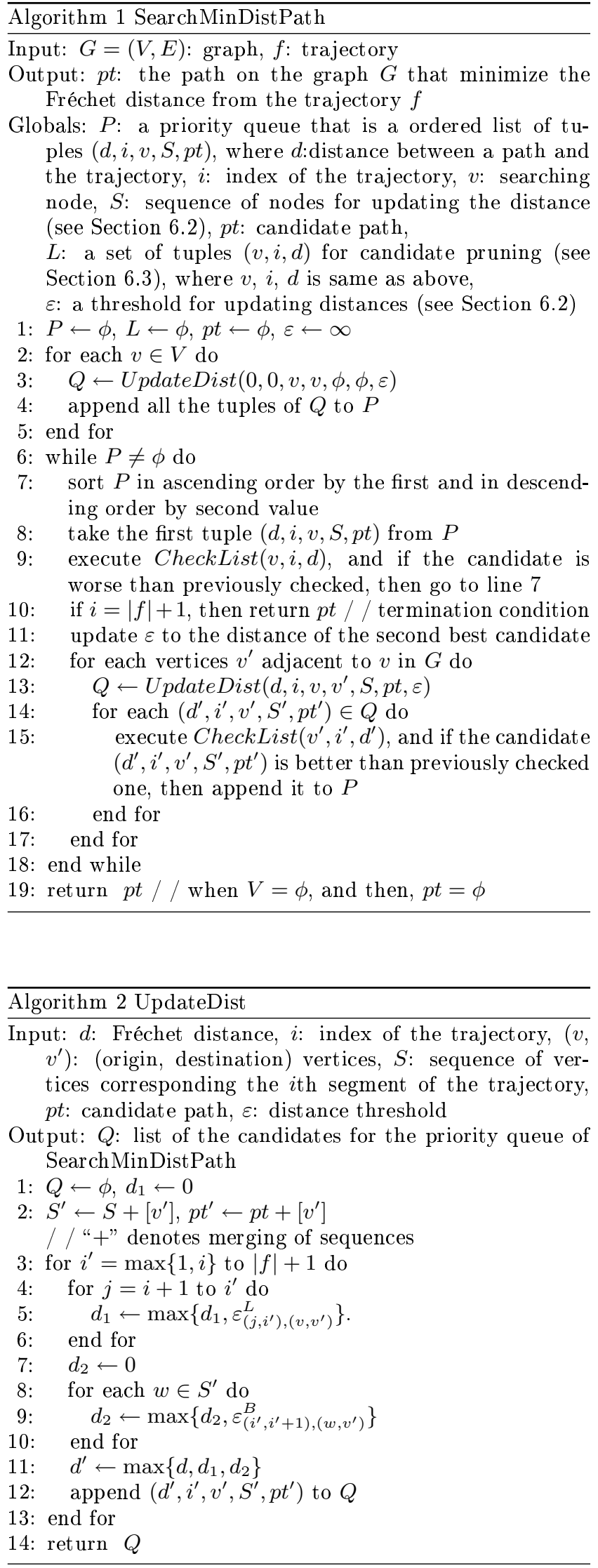

of the trajectory. This increases the number of steps of computation. However, not all of the points correspond to each of the vertices or edges in the graph.

We can optimize algorithm as follows. If the value $d_{1}$ exceeds the threshold $\varepsilon$ before line 7, break the loop of lines $3-13$ and add $Q$ as a candidate. Note that we have to distinguish these candidates from the usual candidates (added in line 12). This makes our algorithm faster for long trajectories.

\subsection{Candidate pruning}

Finally, we present an improvement on the proposed algorithm. During the graph search, we may reach a visited vertex occasionally. If the corresponding Fréchet distance is less than the distance computed when previously visited, its candidate may correspond to a new path with a smaller distance from the trajectory. However, for the other case, there is no possibility to find a better candidate. We can narrow down the candidates by such a possibility, which further can be used to decrease the number of steps of computation.

First, in Algorithm 1, we record a tuple $(v, i, d)$ with vertex $v$, the index of the corresponding point of trajectory $i$, and the Fréchet distance $d$ in the list $L$. Then, in Algorithm 3, we check and update the list. For the input candidate $(v, j, d)$, we check the distance $d$ with the recorded distances $d^{\prime \prime}$ with the same vertex $v$ and the index of trajectory $i$. Then, the function CheckList returns 1 if $d$ is less than $d^{\prime \prime},-1$ if $d$ is greater than $d^{\prime \prime}$, and 0 otherwise. Furthermore, we update the record in the list $L$ if $d$ is less than $d^{\prime \prime}$. We narrow down the candidates (line 15) and skip the computations for the candidates with no possibility (line 9 ).

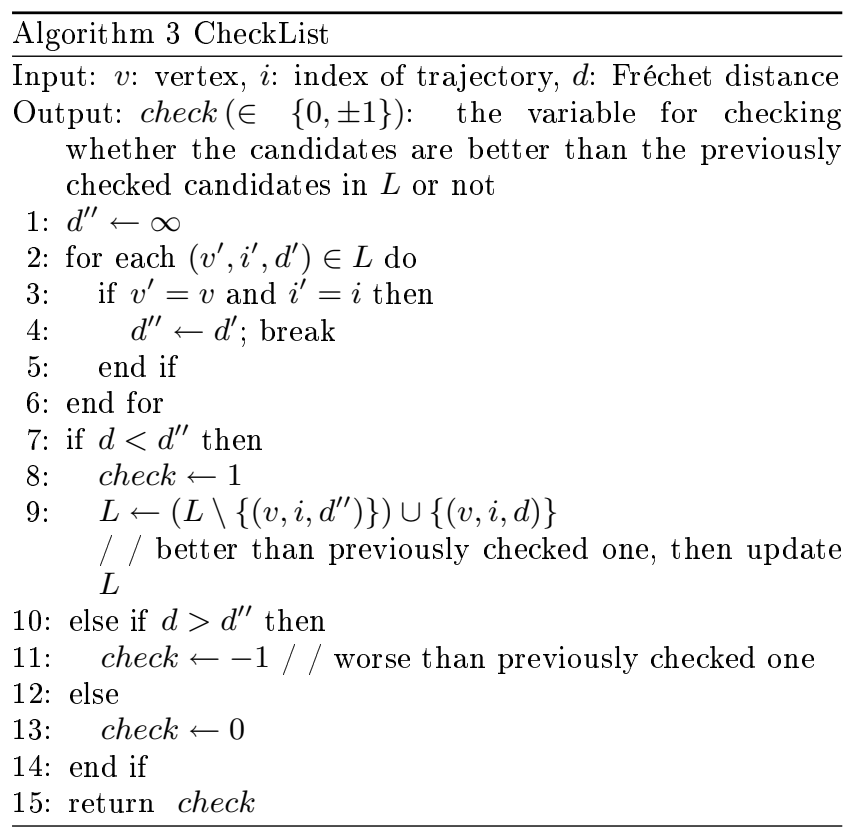

\section{EXPERIMENTAL EVALUATION}

In this section, we present an experimental evaluation to confirm that our algorithm is fast and practical. The experiment was done by using artificial and actual data to 
investigate the computational times of our algorithm with the algorithm of Alt et al. [3].

\subsection{Experiment settings}

For the experiments, we prepared the following four datasets.

ICDM10. The first dataset was published in the IEEE ICDM Contest $^{2}$ in 2010. It contains trajectories from a traffic simulator. In the experiment, we use "Street graph" data as a graph with 19,816 vertices and 35,170 edges and "Training data" as trajectories. From the Training data, we sampled 4,257 trajectories from the first dataset.

T-drive. The second dataset is a sample of a T-drive trajectory dataset published on the $\mathrm{web}^{3}$ (See also [23, 24]). It contains the trajectories of more than ten thousand taxis from February 2nd to the 8th in Beijing. We sampled 5,000 subtrajectories that travel about 20 kilometers in the area defined in Table 1.

For the graph, we used a map from Mapzen ${ }^{4}$, which provides citywide maps extracted from the OpenStreetMap ${ }^{5}$ database. We omitted links with the tags "footway", "cycleway," and so on where the taxis could not drive.

Bikely. The third dataset is one we collected from the Bikely ${ }^{6}$ website. It contains about two hundred thousand trajectories of bicycles around the world collected since 2006. We sampled 3,150 trajectories that travel at least 2 kilometers in the area defined in Table 1. For the graph, we used a map from Mapzen similarly to the T-drive dataset, where we did not omit links with the tags "footway", "cycleway," and so on.

GeoLife. The fourth dataset is GeoLife GPS trajectory dataset published on the web ${ }^{7}$ (See also $[28,29,30]$ ) It contains the trajectories of 182 users in five years in Beijing. We sampled 4,837 subtrajectories that have label "Walk" and travel at most 2 kilometers in the area defined in Table 1. For the graph, we used a map from Mapzen same as the T-drive dataset, where we did not omit links.

Table 1 contains details of each dataset and graph. The Bikely dataset does not have a timestamp of each point, so we could not calculate the travel time and time intervals.

In the table, we can see that the average travel distance of

${ }^{2}$ IEEE ICDM Contest: TomTom traffic prediction for intelligent GPS navigation, URL: http://tunedit.org/challenge/IEEE-ICDM-2010

${ }^{3}$ T-drive: driving directions based on taxi traces, URL: http://research.microsoft.com/apps/pubs/default.aspx?id=138035

${ }^{4}$ Mapzen, URL: https://mapzen.com/metro-extracts/

${ }^{5}$ OpenStreetMaps, URL: http://www.openstreetmap.org/

${ }^{6}$ Bikely, URL: http://www.bikely.com

${ }^{7}$ GeoLife: building social networks using human location history URL: http://research.microsoft.com/enus/projects/geolife/
Table 1: Datasets for the Experiment

\begin{tabular}{|c|c|c|c|c|c|}
\hline \multicolumn{2}{|c|}{ Dataset } & ICDM 10 & T-drive & Bikely & GeoLife \\
\hline \multicolumn{6}{|c|}{ [Area] } \\
\hline \multirow[t]{2}{*}{ lat. } & min. (deg.) & 52.11 & 39.80 & 47.4 & 39.80 \\
\hline & max. (deg.) & 52.38 & 40.10 & 47.8 & 40.10 \\
\hline \multirow[t]{2}{*}{ lon. } & min. (deg.) & 20.83 & 116.20 & -122.45 & 116.20 \\
\hline & max. (deg.) & 21.27 & 116.55 & -122.10 & 116.55 \\
\hline \multicolumn{6}{|c|}{ [Trajectories] } \\
\hline \multicolumn{2}{|c|}{ num. of trajectories } & 4,257 & 5,000 & 3,150 & 4,837 \\
\hline \multicolumn{2}{|c|}{ ave. num. of points } & 672 & 33.4 & 175 & 244 \\
\hline \multicolumn{2}{|c|}{ ave. travel time (min.) } & 112 & 125 & - & 16.2 \\
\hline \multicolumn{2}{|c|}{ ave. travel dist. $(\mathrm{km})$} & 16.6 & 21.4 & 24.3 & 0.911 \\
\hline \multicolumn{2}{|c|}{ ave. time interval (sec.) } & 10.0 & 231 & - & 3.99 \\
\hline \multicolumn{2}{|c|}{ ave. dist. interval (m) } & 24.7 & 661 & 140 & 3.75 \\
\hline \multicolumn{6}{|c|}{ [Graph] } \\
\hline \multicolumn{2}{|c|}{ num. of vertices } & 19,816 & 67,546 & 262,699 & 92,008 \\
\hline \multicolumn{2}{|c|}{ num. of edges } & 35,170 & 110,977 & 540,017 & 163,431 \\
\hline \multicolumn{2}{|c|}{ ave. len. of edges $(\mathrm{m})$} & 113.1 & 94.1 & 38.8 & 73.6 \\
\hline
\end{tabular}

each dataset except for the GoeLife dataset is around twenty kilometers, but the difference of sampling rates and speeds caused variations in the distance intervals.

We performed the experiment with the four algorithms described as follows. As we see in Section 4.2, the algorithm used by Alt et al. [3] has iterations for selecting critical values and executing the algorithm for the decision problem. In this experiment, we investigated the total computational time by using a binary search on a set of candidates of the Fréchet distance (Alt [Binary]) and the computation time of the algorithm for the decision problem in the last computation of the decision problem in the binary search (Alt [Decision]).

For our method, we prepared two algorithms. One is the algorithm described in Section 6 (Ours [Dijkstra]), and the other is the algorithm with the breadth-first search (Ours [Breadth]) instead of the Dijkstra algorithm. This latter algorithm is for comparing the results of the experiment with those of the algorithm described in Alt et al. [3], which follows the breadth-first search algorithm.

All of the algorithms were implemented in Python. All the experiments were carried out on a CentOS 6.4 with Linux kernel 2.6 with an Intel Xeon CPU E5-2690 2.90GHz machine with 768 GB of RAM. The system ran Cygwin and Python 2.7.8.

\subsection{Result}

Table 2 and Figure 8 show the average computation time of each method. Our algorithm (Ours [Dijkstra]) was at least 50 times (the case with the ICDM10 dataset) faster than Alt's algorithm (Alt [Binary]).

Comparing the computational times for each dataset, the times for the Bikely and the GeoLife datasets were larger than those for the other datasets. As we see in Table 1, the average lengths of the edges of the graph for the Bikely and the GeoLife datasets were smaller than those of the other datasets. Additionally, the computational time for the ICDM10 dataset was larger than that for the T-drive 
dataset. The average distance interval of the ICDM10 dataset was smaller than that of the T-drive dataset.

Table 2: Experimental result

\begin{tabular}{|c|c|c|c|c|c|}
\hline \multicolumn{2}{|c|}{ Method } & \multicolumn{4}{c|}{ Time [s] } \\
\cline { 2 - 6 } & ICDM10 & T-drive & Bikely & GeoLife \\
\hline \multirow{2}{*}{ Ours } & {$[$ Dijkstra] } & 11.27 & 7.50 & 19.22 & 36.30 \\
\cline { 2 - 6 } & {$[$ Breadth] } & 27.35 & 11.57 & 31.16 & 103.62 \\
\hline \multirow{2}{*}{ Alt } & {$[$ Decision] } & 59.83 & 234.76 & 233.93 & 112.35 \\
\cline { 2 - 6 } & {$[$ Binary] } & 561.48 & 479.29 & $3,111.52$ & $2,001.39$ \\
\hline
\end{tabular}

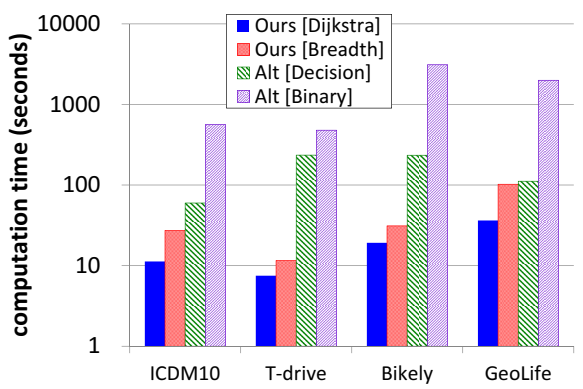

Figure 8: Experimental result: The graph plots the average computation times of each algorithm for each dataset.

\subsection{Scalability}

We also tested scalability of our algorithm (Ours [Dijkstra]) for larger datasets. We focused on three values for scale of the dataset; (1) number of trajectories, (2) average travel distance, and (3) size of graph (number of nodes). The values (1) and (2) are related with the size of trajectory. From T-drive trajectory dataset and OpenStreetMap database, we resampled 15 set of trajectories and graphs defined in Table 3.

Maximum memory usage for each case of (1), (2), and (3) were $5.10 \mathrm{~GB}, 5.07 \mathrm{~GB}$, and $3.51 \mathrm{~GB}$, respectively. For the computation time, Table 3 and Figure 9 shows the experimental results. As the result, our algorithm performs linearly in computation time with the sizes of trajectories and graphs, except for the largest case of (3).

For the case of (3), we sampled the largest graph by choosing large area, which included the suburbs near mountains, where the road network was very sparse. Though the graph is very large, the number of candidate paths for a given trajectory may be small.

\subsection{Discussion}

The main factor in the increase in speed of our algorithm is the reduction in the number of iterations. We designed and proposed an algorithm with only one iteration, although we need some iterations to execute the algorithm for the decision problem in order to determine the Fréchet distance in the algorithm of Alt et al. [3]. In fact, Alt's algorithm (Alt [Binary]) costs much more time than that with one iteration (Alt [Decision]).
Table 3: Datasets for scalability and results (1) Average travel distance: $21.4 \mathrm{~km}$, number of node: 67,546 .

\begin{tabular}{c|ccccc}
\hline num. trajectories $(\mathrm{K})$ & 10 & 20 & 30 & 40 & 50 \\
\hline total comp. time (h) & 23.6 & 46.4 & 73.9 & 103 & 132
\end{tabular}

(2) Number of trajectories: 10,000 , number of node: 67,546 . \begin{tabular}{c|ccccc}
\hline ave. travel dist. $(\mathrm{km})$ & 11.4 & 21.4 & 31.4 & 41.5 & 51.4 \\
\hline ave. comp. time $(\mathrm{s})$ & 6.04 & 8.50 & 15.7 & 17.8 & 28.9
\end{tabular}

(3) Number of trajectories: 10,000 , average travel distance: $21.4 \mathrm{~km}$.

\begin{tabular}{c|ccccc}
\hline num. of nodes $(\mathrm{K})$ & 35.6 & 67.5 & 106 & 139 & 175 \\
\hline ave. comp. time $(\mathrm{s})$ & 6.63 & 8.50 & 11.8 & 16.1 & 14.2 \\
\hline
\end{tabular}

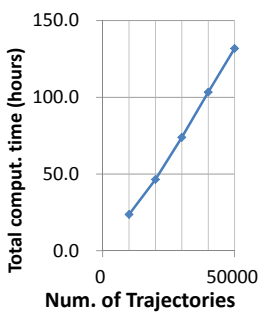

(1)

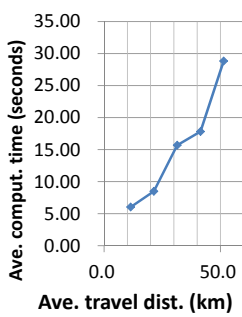

(2)

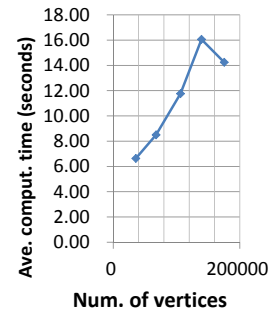

(3)
Figure 9: Experimental result [scalability]: The graph plots the computation times for each dataset.

Note that we can use parametric search [9] instead of binary search in the algorithm of Alt et al. [3]. Actually, experimental results by van Oostrum et al. [20] shows the algorithm with parametric search and quicksort is faster than that with binary search for the optimization problem solving the Fréchet distance of curves. However, since some iterations are needed in their architecture, the total computation time must be greater than that of the last iteration. Practically, we find that our algorithm is at least four times faster than the last iteration (Alt [Decision]).

In addition, our algorithm was a Dijkstra-type algorithm, where we compute and update distances only for the prior candidate in each step; thus, we do not have to compute distances for all candidates except for in the worst case scenarios. Additionally, the algorithm of Alt et al. [3] uses a breadth-first search, which is the same as Ours [Breadth]. As was seen from the comparison between the experimental results of Ours [Dijkstra] and Ours [Breadth], the Dijkstra algorithm was effective at speeding up overall computations.

From another point of view, by solving the optimization problem on a graph, we obtain a path on the graph and its Fréchet distance from a given trajectory. Then, we can also solve the decision problem on a graph by judging if the distance is larger than a given threshold or not. Our algorithm (Ours [Dijkstra]) was faster than the algorithm of the decision problem of Alt et al. [3] (Alt [Decision]). These results suggest that the optimization problem is superior to the decision problem with respect to speed. 


\section{CONCLUSION}

We proposed a fast and practical algorithm for the optimization problem on a graph that searches a path on a given graph that minimizes the Fréchet distance from a given trajectory. We proposed a Dijkstra-type algorithm with a priority that is the Fréchet distance between a candidate path and the corresponding part of the trajectory. Experimental results show that our algorithm was more than fifty times faster than that of Alt et al. [3], which uses the binary search method.

In the future, we will work on the development of trajectoryanalysis technologies, which can be used to recommend destinations or predict travel times. For this, we will use wellcorrected trajectory data obtained with our map-matching method or we will consolidate similar trajectories.

\section{REFERENCES}

[1] M. Ahmed and C. Wenk: Constructing street networks from GPS trajectories, In: Proc. 20th ESA, LNCS 7501, 60-71, 2012.

[2] H. Alt and M. Godau: Computing the Fréchet distance between two polygonal curves, Internat. J. Comput. Geom. Appl., 5, 75-91, 1995.

[3] H. Alt, A. Efrat, G. Rote, and C. Wenk: Matching planar maps, J. Algorithms 49, 262-283, 2003.

[4] H. Alt, C. Knauer, and C. Wenk: Comparison of distance measure for planar curves, Algorithmica 38, 45-58, 2004.

[5] D. Bernstein and A. Kornhauser: An introduction to map matching for personal navigation assistants, Technical report, New Jersey TIDE Center, 1996.

[6] S. Brakatsoulas, D. Pfoser, R. Salas, and C. Wenk: On map-matching vehicle tracking data, In: Proc. 31st VLDB, 853-864, 2005.

[7] K. Buchin, M. Buchin, J. Gudmundsson, M. Löffler and J. Luo: Detecting commuting patterns by clustering subtrajectories, In: Proc. 19th ISAAC, LNCS 5369, 644-655, 2008.

[8] L. Cao and J. Krumm: From GPS traces to a routable road map, In: Proc. 17th ACM SIGSPATIAL GIS, $3-12,2009$.

[9] R. Cole: Slowing down sorting networks to obtain faster sorting algorithms, J. Assoc. Comput. Mach., 34, 200-208, 1987.

[10] T. H. Cormen, C. E. Leiserson, R. L. Rivest, and C. Stein: Introduction to algorithms (2nd ed.), The MIT Press and McGraw-Hill, 2001.

[11] G. Derekenaris, J. Garofalakis, C. Makris, J. Prentzas, S. Sioutas and A. Tsakalidis: Integrating GIS, GPS and GSM technologies for the effective management of ambulances, Comput. Environ. Urban Systems, 25, 267-278, 2001.

[12] A. Driemel, S. Har-Peled, and C. Wenk: Approximating the Fréchet distance for realistic curves in near linear time, Discrete Comput. Geom., 48, 94-127, 2012.

[13] M. M. Fréchet: Sur quelques points du calcul fonctionnel, Rend. Circ. Mat. Palermo, 22, 1-74, 1906.

[14] H. Gonzalez, J. Han, X. Li, M. Myslinska, and J. P. Sondag: Adaptive fastest path computation on a road network: A traffic mining approach, In: Proc. 33rd
VLDB, 794-805, 2007.

[15] J. S. Greenfeld: Matching GPS observations to location on a digital map, In: Proc. 81st Annual Meeting TRB (CD-ROM), 2002.

[16] Y. Li, Q. Hung, M. Kerber, L. Zhang, and L. Guibas: Large-scale joint map matching of GPS traces, In: 21st ACM SIGSPATIAL GIS, 214-223, 2013.

[17] Y. Lou, C. Zhang, Y. Zheng, X. Xie, W. Wang, and Y. Huang: Map-matching for low-sampling-rate GPS trajectories, In: Proc. 17th ACM SIGSPATIAL GIS, $352-361,2009$.

[18] A. Mosig and M. Clausen: Approximately matching polygonal curves with respect to the Fréchet distance, Comput. Geom., 30, 113-127, 2005.

[19] P. Newson and J. Krumm: Hidden Markov map matching through noise and sparseness, In: Proc. 17th ACM SIGSPATIAL GIS, 336-343, 2009.

[20] R. van Oostrum and R. C. Veltkamp: Parametric search made practical, Comput. Geom., 28, 75-88, 2004.

[21] D. Pfoser and C. S. Jensen: Capturing the uncertainty of moving-object representations, In: Proc. 6th SSD, LNCS 1651, 111-131, 1999.

[22] M. A. Quddus, W. Y. Ochieng, L. Zhao, and R. B. Noland: A general map matching algorithm for transport telematics applications, GPS Sol., 7, 157-167, 2003.

[23] J. Yuan, Y. Zheng, C. Zhang, W. Xie, G. Sun, and Y. Huang: T-drive: driving directions based on taxi trajectories, In: Proc. 18th ACM SIGSPATIAL GIS, 99-108, 2010

[24] J. Yuan, Y. Zheng, X. Xie, and G. Sun: Driving with knowledge from the physical world, In: Proc. 17th ACM SIGKDD KDD, 316-324, 2011.

[25] C. Wenk, R. Salas, and D. Pfoser: Addressing the need for map-matching speed: localizing global curve-matching algorithms, In: Proc. 18th SSDBM, 379-388, 2006.

[26] C. E. White, D. Bernstein, and A. L. Kornhauser: Some map matching algorithms for personal navigation assistants, Transportation Research Part C, 8, 91-108, 2000.

[27] D. Yoshida, X. Song, and V. Raghavan: Development of track log and point of interest management system using free and open source software, Appl. Geomat., 2, 123-135, 2010.

[28] Yu Zheng, Quannan Li, Yukun Chen, Xing Xie, Wei-Ying Ma: Understanding mobility based on GPS data, In: Proc. 10th UbiComp, 312-321, 2008.

[29] Yu Zheng, Lizhu Zhang, Xing Xie, Wei-Ying Ma: Mining interesting locations and travel sequences from GPS trajectories, In: Proc. 18th WWW, 791-800, 2009.

[30] Yu Zheng, Xing Xie, Wei-Ying Ma: GeoLife: A collaborative social networking service among user, location and trajectory, IEEE Data Eng. Bull., 33, 32-40, 2010.

[31] H. Zhu, J. Luo, H. Yin, X. Zhou, J. Z. Huang, and F. B. Zhan: Mining trajectory corridors using Fréchet distance and meshing grids, In: Proc. 14th PAKDD Part I, LNAI 6118, 228-237, 2010. 